Acta Crystallographica Section F

Structural Biology

and Crystallization

Communications

ISSN 1744-3091

\section{Anneleen Vuchelen, ${ }^{a, b}$ Els Pardon, ${ }^{a, b}$ Jan Steyaert, ${ }^{\text {a,b }}$ Benoît Gamain, ${ }^{c, d, e, f}$ Remy Loris, ${ }^{a, b}$ * Nico A. J. van Nuland ${ }^{a, b}$ and Stéphanie Ramboarina ${ }^{a, b *} \neq$}

\footnotetext{
${ }^{\text {a }}$ Structural Biology Brussels, Department of Biotechnology (DBIT), Vrije Universiteit Brussel, Pleinlaan 2, B-1050 Brussels, Belgium,

bolecular Recognition Unit (MoRe), Department of Structural Biology, VIB, Pleinlaan 2, B-1050 Brussels, Belgium, ' Inserm, UMRS665, F-75015 Paris, France, 'Université Paris Diderot, F-75013 Paris, France, e Institut National de la Transfusion Sanguine, F-75015 Paris, France, and 'Laboratoire d'Excellence Gr-Ex, France
}

₹ Present address: Laboratoire de Biologie Structurale et Radiobiologie, Institut de Biologie et de Technologies de Saclay, Direction des Sciences du Vivant, Commissariat à l'Energie Atomique Saclay, 91191 Gif-sur-Yvette CEDEX, France.

Correspondence e-mail: reloris@vub.ac.be, stephanie.ramboarina@cea.fr

Received 23 October 2012 Accepted 18 January 2013

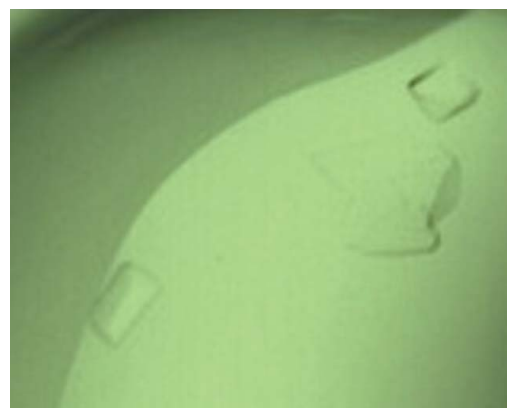

C 2013 International Union of Crystallography All rights reserved

\section{Production, crystallization and X-ray diffraction analysis of two nanobodies against the Duffy binding-like (DBL) domain DBL6e-FCR3 of the Plasmodium falciparum VAR2CSA protein}

The VAR2CSA protein has been closely associated with pregnancy-associated malaria and is recognized as the main adhesin exposed on the surface of Plasmodium falciparum-infected erythrocytes. Chondroitin sulfate A was identified as the main host receptor in the placenta. Single-domain heavy-chain camelid antibodies, more commonly called nanobodies, were selected and produced against the DBL6 $\varepsilon$-FCR3 domain of VAR2CSA. Crystals of two specific nanobodies, Nb2907 and Nb2919, identified as strong binders to DBL6عFCR3 and the full-length VAR2CSA exposed on the surface of FCR3 $P$. falciparum-infected erythrocytes, were obtained. Crystals of $\mathrm{Nb} 2907$ diffract to $2.45 \AA$ resolution and belong to space group $C 2$ with unit-cell parameters $a=136.1, b=78.5, c=103.4 \AA, \beta=118.8^{\circ}$, whereas $\mathrm{Nb} 2919$ crystals diffract to $2.15 \AA$ resolution and belong to space group $P 4_{3} 2{ }_{1} 2$ with unit-cell parameters $a=b=62.7, c=167.2 \AA$.

\section{Introduction}

Pregnancy-associated malaria is correlated to the cyto-adhesion of Plasmodium falciparum-infected erythrocytes (PEs) to the placenta causing up to 200000 foetal and infant deaths and at least 10000 maternal deaths per year. The $P$. falciparum VAR2CSA protein was identified as the key adhesin responsible for the sequestration of PEs into the placenta through its interaction with chondroitin sulfate A (CSA) (Salanti et al., 2004). VAR2CSA is a $350 \mathrm{kDa}$ protein composed of six cysteine-rich Duffy binding-like (DBL) domains. Small-angle X-ray scattering experiments showed these domains are folded into a compact structure forming a specific and high-affinity binding site for CSA (Srivastava et al., 2010). VAR2CSA has been shown to become the target of maternal antibodies after subsequent pregnancies (Fried et al., 1998; Salanti et al., 2004). An evasion of immunity to placental malaria would imply the masking of VAR2CSA-specific IgG epitopes by IgM antibodies. The C-terminal part of VAR2CSA, including DBL6 $\varepsilon$, would be involved in IgM recognition (Rasti et al., 2006; Barfod et al., 2011).

Nanobodies are constituted of the single VHH domain of camelid heavy-chain antibodies. They are small and stable monomeric proteins $(14 \mathrm{kDa})$ displaying a high affinity for their antigen and have been shown to be efficient tools for the crystallization of difficult systems (Baranova et al., 2012; Steyaert \& Kobilka, 2011) and for the characterization of biological mechanisms (Domanska et al., 2011).

Nanobodies against the DBL6 $\varepsilon$ domain of the VAR2CSA protein from the FCR3 $P$. falciparum strain were produced to help decipher the role of DBL6 $\varepsilon$ in VAR2CSA adhesion to the placenta and evasion of the host immune response. With this aim in mind, structural and biophysical studies of nanobodies in complex with the DBL6 $\varepsilon$ domain were initiated in order to describe at the atomic level the receptor binding site or potential epitopes for the host immune system. As part of our ongoing studies, we here present the crystallization of two specific nanobodies able to recognize the isolated DBL6 $\varepsilon$-FCR3 domain and VAR2CSA-FCR3 exposed on infected erythrocytes. This implies that the epitope of these nanobodies is available in the compact structure formed by the full-length VAR2CSA. 


\section{Material and methods}

\subsection{Expression and purification of DBL6 $\varepsilon$-FCR3}

The DNA sequence coding for DBL6 6 -FCR3 was cloned into a pET15b expression vector that introduces a His tag consisting of six histidines at the $\mathrm{N}$-terminus of the protein. The vector was transformed into the Escherichia coli Rosetta gami strain, which is specifically designed for proteins containing multiple cysteines.

$500 \mathrm{ml}$ of $25 \mathrm{mg} \mathrm{ml}^{-1} \mathrm{LB}$ containing $100 \mu \mathrm{g} \mathrm{ml}^{-1}$ ampicillin and $25 \mu \mathrm{g} \mathrm{ml}^{-1}$ chloramphenicol was inoculated with $10 \mathrm{ml}$ of preculture and left to grow overnight at $310 \mathrm{~K}$ under shaking. When the culture reached an $\mathrm{OD}_{600}$ of $1.0,0.1 \mathrm{~m} M$ IPTG was added and the temperature was decreased to $289 \mathrm{~K}$ to allow the overnight expression of soluble protein. The cells were harvested the next day by centrifugation for $15 \mathrm{~min}$ at $6490 \mathrm{~g}$. The pellet was resuspended in $40 \mathrm{ml}$ of $50 \mathrm{~m} M$ phosphate buffer $\mathrm{pH} 6.3,50 \mathrm{~m} M$ cystamine and the cells were broken by sonicating three times for $5 \mathrm{~min}$ at $275 \mathrm{~K}$. The solution obtained was centrifuged for $30 \mathrm{~min}$ at $12096 \mathrm{~g}$. The supernatant was loaded onto a $1 \mathrm{ml} \mathrm{Ni-NTA} \mathrm{column} \mathrm{(HisTrap} \mathrm{HP,} \mathrm{GE}$ Healthcare) equilibrated in $50 \mathrm{~m} M$ phosphate buffer $\mathrm{pH} 6.3,100 \mathrm{~m} M$ $\mathrm{NaCl}, 30 \mathrm{~m} M$ imidazole, $50 \mathrm{~m} M$ cystamine. DBL6 $\varepsilon$-FCR3 was eluted using $50 \mathrm{~m} M$ phosphate buffer $\mathrm{pH} 6.3,50 \mathrm{~m} M \mathrm{NaCl}, 400 \mathrm{~m} M$ imidazole, $50 \mathrm{~m} M$ cystamine. The eluted protein was subsequently loaded onto a Superdex 75 16/60 column (GE Healthcare) equilibrated in $50 \mathrm{~m} M$ phosphate buffer $\mathrm{pH} 7.0,10 \mathrm{~m} M$ cystamine and eluted using the same buffer.

\subsection{Generation of nanobodies against DBL6e}

Recombinant DBL6 $\varepsilon$-FCR3 was injected subcutaneously every $7 \mathrm{~d}$ for 5 weeks in a Lama glama at a concentration of $0.44 \mathrm{mg} \mathrm{ml}^{-1}$ in $50 \mathrm{~m} M$ phosphate buffer $\mathrm{pH}$ 7.0, $10 \mathrm{~m} M$ cystamine. Screening and selection of nanobodies were performed as previously described (Conrath et al., 2005). Briefly, lymphocytes were purified from the blood sample obtained 1 week after the last immunization. cDNA was synthesized from the acquired RNA using a reverse PCR protocol. A VHH phage display library was constructed using the pMES4 expression and phagemid vector. This vector introduces a pelB signal peptide and a C-terminal His tag consisting of six histidines. Multiple rounds of panning and screening of this library resulted in eight binders to DBL6 $\varepsilon$-FCR3. These nanobodies of interest, which are still in the pMES4 vector, were subsequently transformed into the E. coli WK6 expression strain.

\subsection{Purification of nanobodies}

For nanobody production, a preculture was grown at $310 \mathrm{~K}$ with shaking in $25 \mathrm{mg} \mathrm{ml}^{-1} \mathrm{LB}$ containing $100 \mu \mathrm{g} \mathrm{ml}^{-1}$ ampicillin, $2 \%$ glucose and $1 \mathrm{mM} \mathrm{MgCl} 2.11$ of TB (Terrific Broth) supplemented with the same reagents was inoculated with $10 \mathrm{ml}$ preculture. $1 \mathrm{mM}$ IPTG was added once the $\mathrm{OD}_{600}$ reached 0.7 . The induced cultures were left overnight for protein expression and secretion to the periplasm at $301 \mathrm{~K}$ with shaking. The cells were harvested by centrifugation for $15 \mathrm{~min}$ at $6490 \mathrm{~g}$. The pellet was resuspended in $15 \mathrm{ml}$ of $200 \mathrm{~m} M$ Tris- $\mathrm{HCl} \mathrm{pH} 8.0,500 \mathrm{~m} M$ EDTA and $500 \mathrm{~m} M$ sucrose for $1 \mathrm{~h}$ under stirring on ice. An osmotic shock was subsequently applied by addition of $30 \mathrm{ml}$ of $50 \mathrm{mM}$ Tris- $\mathrm{HCl} \mathrm{pH} 8.0$, $125 \mathrm{~m} M$ EDTA and $125 \mathrm{~m} M$ sucrose and left for $1 \mathrm{~h}$ under stirring on ice. After centrifugation for $30 \mathrm{~min}$ at $12096 \mathrm{~g}$ the pellet was discarded and the periplasmic extract was loaded onto a $1 \mathrm{ml} \mathrm{Ni}-$ NTA column (HisTrap HP, GE Healthcare) equilibrated in $50 \mathrm{mM}$ phosphate buffer $\mathrm{pH} 7.0,1 \mathrm{M} \mathrm{NaCl}$ and washed with $50 \mathrm{~m} M$ phosphate buffer $\mathrm{pH} 6.0,1 \mathrm{M} \mathrm{NaCl}$. The nanobodies were eluted using $50 \mathrm{~m} M$ phosphate buffer $\mathrm{pH} 7.0,1 \mathrm{M} \mathrm{NaCl}, 0.5 \mathrm{M}$ imidazole and loaded onto a Superdex 75 16/60 column (GE Healthcare) preequilibrated in $50 \mathrm{~m} M$ Tris- $\mathrm{HCl}$ pH 7.5, $150 \mathrm{mM} \mathrm{NaCl}$.

\subsection{Crystallization}

Crystallization trials were initiated for nanobodies that are able to recognize both the DBL6 $\varepsilon$-FCR3 domain and the full-length VAR2CSA-FCR3 exposed on the surface of P. falciparum-infected red blood cells, which implies their epitope is still available in the compact VAR2CSA. The purified nanobodies, which still contain their His tag, were concentrated in $50 \mathrm{~m} M$ Tris- $\mathrm{HCl} \mathrm{pH} \mathrm{7.5,} 150 \mathrm{mM}$ $\mathrm{NaCl}$ using an ultrafiltration unit (3000 Da cutoff, Centricon). Crystallization conditions for two nanobodies, Nb2907 and Nb2919, were obtained using a Phoenix robot in 96-well Intelli-Plates (Art Robbins Instruments) with commercial screens from Hampton Research (Crystal Screen and Crystal Screen Cryo) and Molecular Dimensions (Proplex, JCSG-plus and Morpheus). The sitting-drop vapourdiffusion method was used with drops consisting of $100 \mathrm{nl}$ nanobody sample mixed with an equal amount of screening solution. $70 \mu \mathrm{l}$ of the screening solution were placed into the reservoir of the plates and the plates were stored at $292 \mathrm{~K}$.

\subsection{Data collection and analysis}

Crystals of the two nanobodies were directly mounted from the drops in a cryoloop and flash-cooled in the nitrogen-gas cryostream without additional cryoprotectant. A complete diffraction data set was collected from one crystal of Nb2907 on PROXIMA-1 of the SOLEIL synchrotron (Gif-sur-Yvette, France) using a PILATUS 6M detector (see Table 1). 1000 images were collected with an oscillation step of $0.2^{\circ}$ and $0.4 \mathrm{~s}$ exposure time. The data were integrated, scaled and merged using the XDS package (Kabsch, 2010). The merged intensities were converted to structure-factor amplitudes using the program TRUNCATE from the CCP4 package (Winn et al., 2011).

For nanobody Nb2919 data were recorded for several crystals of Nb2919 on beamline X06DA (SLS, Villigen, Switzerland) using a MAR Mosaic CCD detector (see Table 1). The data set considered to be the best consists of 120 images measured using an oscillation step of $1^{\circ}$ and $1.0 \mathrm{~s}$ exposure time. Data were integrated using $i M O S F L M$

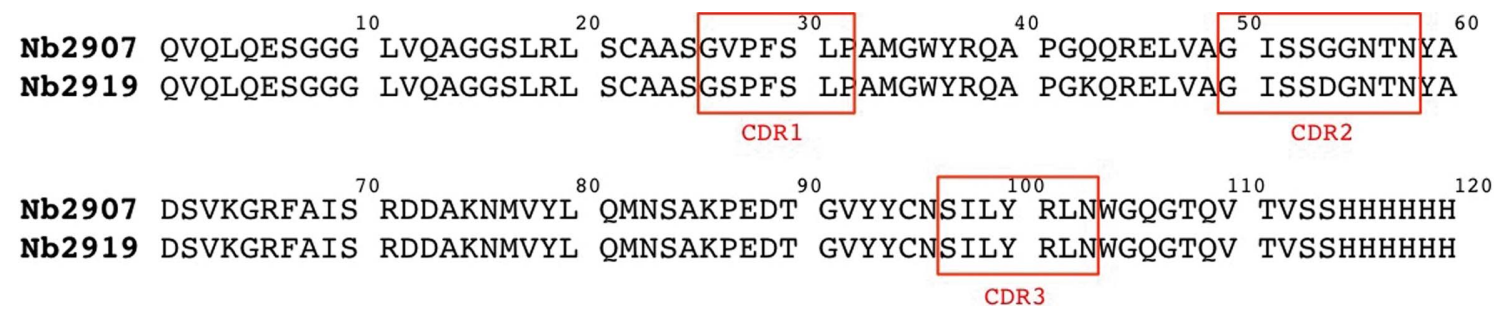

Figure 1

CDR3

Sequence alignment of Nb2907 and Nb2919. The complementary determining regions (CDR) are shown in red boxes. 
Table 1

Data-collection statistics.

Values for the outer shell are given in parentheses.

\begin{tabular}{|c|c|c|}
\hline & $\mathrm{Nb} 2907$ & $\mathrm{Nb} 2919$ \\
\hline Diffraction source & PROXIMA-1 (SOLEIL, France) & X06DA (SLS, Switzerland) \\
\hline Wavelength (§) & 0.98 & 1.00 \\
\hline Detector & PILATUS $6 \mathrm{M}$ & MAR Mosaic CCD \\
\hline Crystal-detector distance ( $\mathrm{mm})$ & 509.1 & 199.8 \\
\hline Space group & $C 2$ & $P 4_{3} 2_{1} 2$ \\
\hline Unit-cell dimensions $\left(\AA,^{\circ}\right)$ & $a=136.1, b=78.5, c=103.4, \alpha=90, \beta=118.8, \gamma=90$ & $a=62.7, b=62.7, c=167.2, \alpha=\beta=\gamma=90$ \\
\hline Mosaicity $\left({ }^{\circ}\right)$ & 0.31 & 0.75 \\
\hline Resolution range $(\AA)$ & $47.46-2.45(2.58-2.45)$ & $34.78-2.15(2.27-2.15)$ \\
\hline$R_{\text {merge }} \dagger$ & $0.097(0.50)$ & $0.078(0.37)$ \\
\hline 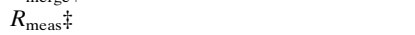 & $0.10(0.60)$ & $0.09(0.44)$ \\
\hline Total No. of reflections & $125228(16670)$ & $59781(8464)$ \\
\hline No. of unique reflections & $34820(4969)$ & $18702(2667)$ \\
\hline Completeness $(\%)$ & $98.9(98.1)$ & $98.9(98.8)$ \\
\hline Redundancy & $3.6(3.4)$ & $3.2(3.2)$ \\
\hline$\langle I / \sigma(I)\rangle$ & $11.0(2.3)$ & $8.1(2.4)$ \\
\hline Overall $B$ factor from Wilson plot $\left(\AA^{2}\right)$ & 54.1 & 35.8 \\
\hline
\end{tabular}

$\dagger R_{\text {merge }}=\sum_{h k l} \sum_{i}\left|I_{i}(h k l)-\langle I(h k l)\rangle\right| / \sum_{h k l} \sum_{i} I_{i}(h k l) . \quad \ddagger R_{\text {meas }}=\sum_{h k l}\{N(h k l) /[N(h k l)-1]\}^{1 / 2} \sum_{i}\left|I_{i}(h k l)-\langle I(h k l)\rangle\right| / \sum_{h k l} \sum_{i} I_{i}(h k l)$.

(Battye et al., 2011) and subsequently scaled and merged using $S C A L A$ from the CCP4 package (Winn et al., 2011). Reduction to structure-factor amplitudes was performed with TRUNCATE. Matthews coefficients were calculated with the program MATTHEWS_COEF (Winn et al., 2011; Matthews, 1968). Molecularreplacement calculations were carried out with Phaser (McCoy et al., 2007) using PDB entry 1yc7 (83\% sequence identity, Conrath et al., 2005) from which the CDR (complementary determining region) loops were removed. Self-rotation functions were calculated with MOLREP (Vagin \& Teplyakov, 1997) using a Patterson integration radius of $20 \AA$ and a resolution cutoff of $10.0-3.0 \AA$.

\section{Results and discussion}

Nanobodies were generated against the DBL6 6 -FCR3 domain of the $P$. falciparum VAR2CSA protein and strong binders were identified

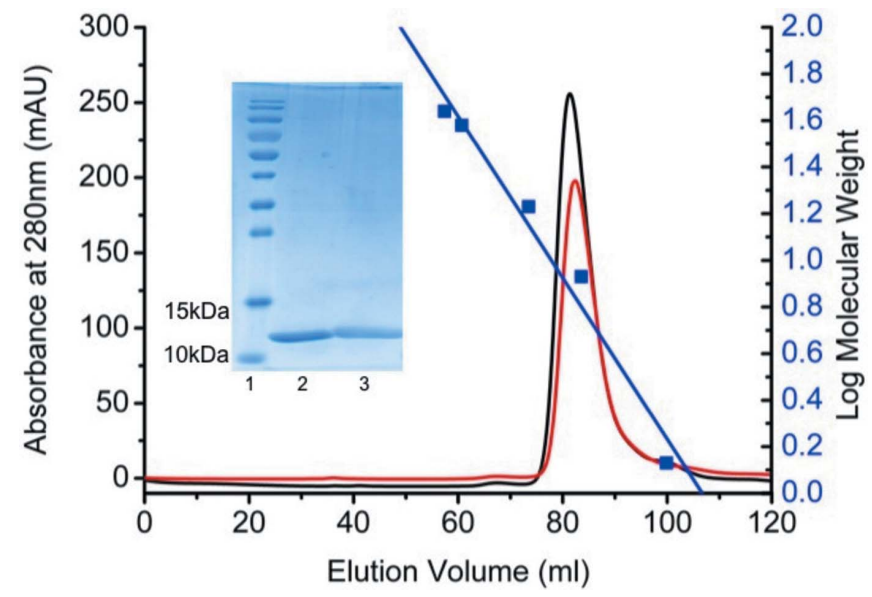

\section{Figure 2}

Nanobody production. Size-exclusion chromatography elution profile of the recombinant Nb2907 (red) and Nb2919 (black) on a Superdex HR 16/60 column equilibrated with a buffer consisting of $50 \mathrm{~m} M$ Tris- $\mathrm{HCl} \mathrm{pH} \mathrm{7.5,150} \mathrm{m} M \mathrm{NaCl}$. The blue squares represent the standard used to determine the relationship between elution volume and molecular weight and consists of vitamin $B_{12}(1.35 \mathrm{kDa})$, ubiquitin (8.6 kDa), myoglobin ( $17 \mathrm{kDa})$, DBL6 $\varepsilon$ (38 kDa) and ovalbumin (44 kDa). Inset: SDS-PAGE of PageRuler Prestained Protein Ladder (lane 1, Thermo Scientific), purified Nb2907 (lane 2) and Nb2919 (lane 3) on 15\% SDSPAGE. by ELISA. These latter have been found to be equally active against the full-length VAR2CSA exposed on infected erythrocytes (results
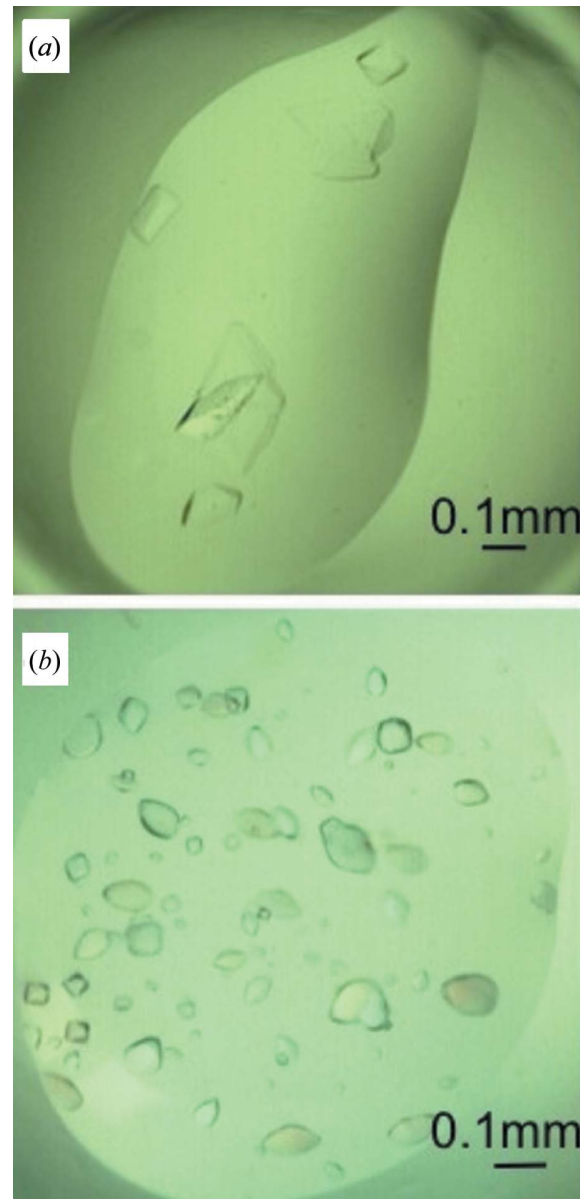

Figure 3

Nanobody crystals. (a) Crystals of Nb2907 grown in 0.1 $M$ HEPES pH 7.5, 4.3 $M$ $\mathrm{NaCl}$. (b) Crystals of Nb2919 grown in 0.1 $M$ HEPES pH 7.5, $0.1 M \mathrm{NaCl}, 1.6 M$ ammonium sulfate. All crystals were grown in 96-well Intelli-Plates (Art Robbins Instruments) (see $\$ 2$ for further details). 
not shown). Two nanobodies against DBL6e-FCR3, Nb2907 and $\mathrm{Nb} 2919$, were crystallized. The amino-acid sequences of nanobodies $\mathrm{Nb} 2907$ and $\mathrm{Nb} 2919$ are 98\% identical (Fig. 1) and both nanobodies are thus expected to bind the same epitope. Preliminary isothermal titration calorimetry (ITC) experiments (using Nb2907 and Nb2919) indicate affinities of 30 and $20 \mathrm{n} M$, respectively, for DBL6 $\varepsilon$-FCR3. Both nanobodies were purified to homogeneity and behave as monomers in size-exclusion chromatography (Fig. 2).

Crystals of $\mathrm{Nb} 2907$ (Fig. $3 a$ ) grew after 1 month from a protein solution of $25 \mathrm{mg} \mathrm{ml}^{-1}(50 \mathrm{~m} M$ Tris- $\mathrm{HCl} \mathrm{pH} \mathrm{7.5,150} \mathrm{mM} \mathrm{NaCl})$ and a precipitant solution consisting of $0.1 M$ HEPES pH 7.5, 4.3 $M \mathrm{NaCl}$. The size of the crystals is $0.08 \times 0.06 \times 0.04 \mathrm{~mm}$. The crystals diffract to $2.45 \AA$ resolution (Fig. $4 a$ ) and belong to space group $C 2$ with unit-

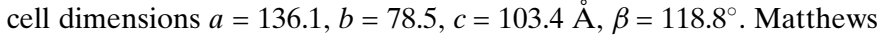
coefficient analysis indicates that the number of nanobody molecules present in the asymmetric unit most likely lies between six and eight,
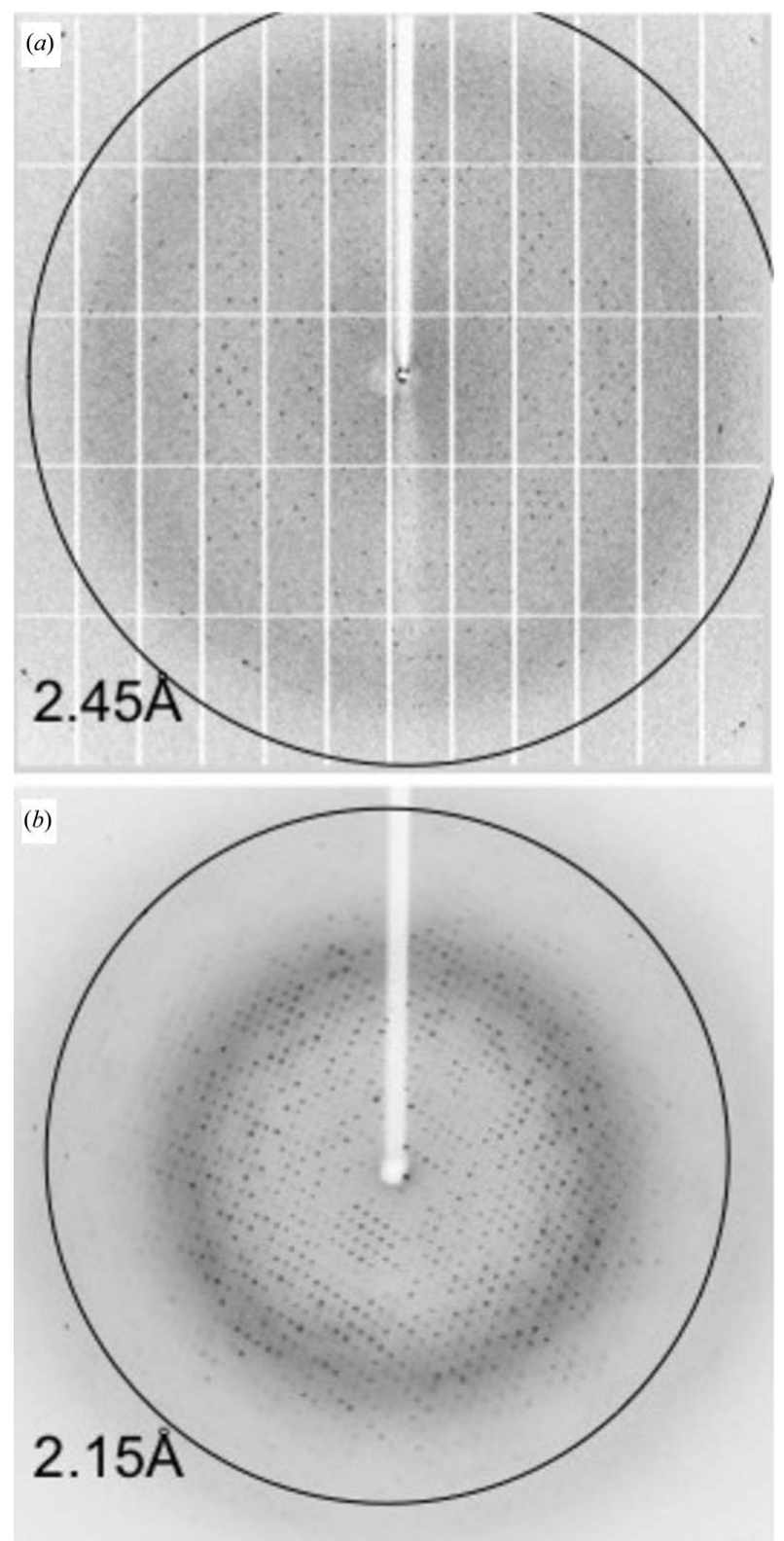

Figure 4

Diffraction pattern collected at $100 \mathrm{~K}$ from the single crystals of $(a) \mathrm{Nb} 2907$ and $(b)$ $\mathrm{Nb} 2919$ that were used for data collection. The maximum resolution is in each case indicated by a circle. See $\$ 2$ for further details. corresponding to Matthews coefficients of 2.88 and $2.16 \AA^{3} \mathrm{Da}^{-1}$ and solvent contents between 57.3 and $43.1 \%$, respectively (combined probability 0.84 ), although there is a small probability for five or nine molecules as well. Molecular replacement using PDB entry 1yc7 as a search model identified six molecules in the asymmetric unit [log likelihood gain (LLG) of 784 for the six-molecule solution compared to 644 and 640 for searches for five and seven molecules, respectively, and a translation function $Z$-score of 21.5 compared to 5.1 for a seventh molecule]. The molecules are packed such that a hexamer is formed consisting of three pairs of $\mathrm{Nb} 2907$ molecules that pack with their $\beta$-sheets upon each other. These pairs then pack with their CDR loops facing each other around a non-crystallographic threefold axis oriented perpendicular to the $a-b$ plane of the unit cell (Fig. 5). This local threefold axis is observed in the self-rotation function as a peak with height $80 \%$ of the crystallographic twofold axis and located at $\theta=0^{\circ}, \varphi=0^{\circ}, \chi=120^{\circ}$.

Crystals of $\mathrm{Nb} 2919$ (Fig. 3b) observable after $4 \mathrm{~d}$ from a protein solution of $23 \mathrm{mg} \mathrm{ml}^{-1}(50 \mathrm{~m} M$ Tris- $\mathrm{HCl} \mathrm{pH} \mathrm{7.5,150} \mathrm{mM} \mathrm{NaCl})$ and a precipitant solution consisting of $0.1 M$ HEPES pH 7.5, $0.1 M \mathrm{NaCl}$, 1.6 $M$ ammonium sulfate. The size of the crystals is $0.09 \times 0.06 \times$ $0.06 \mathrm{~mm}$. The crystals belong to space group $P 4_{3} 2{ }_{1} 2$ with unit-cell dimensions $a=b=62.7, c=167.2 \AA$ and diffract up to $2.15 \AA$ reso-
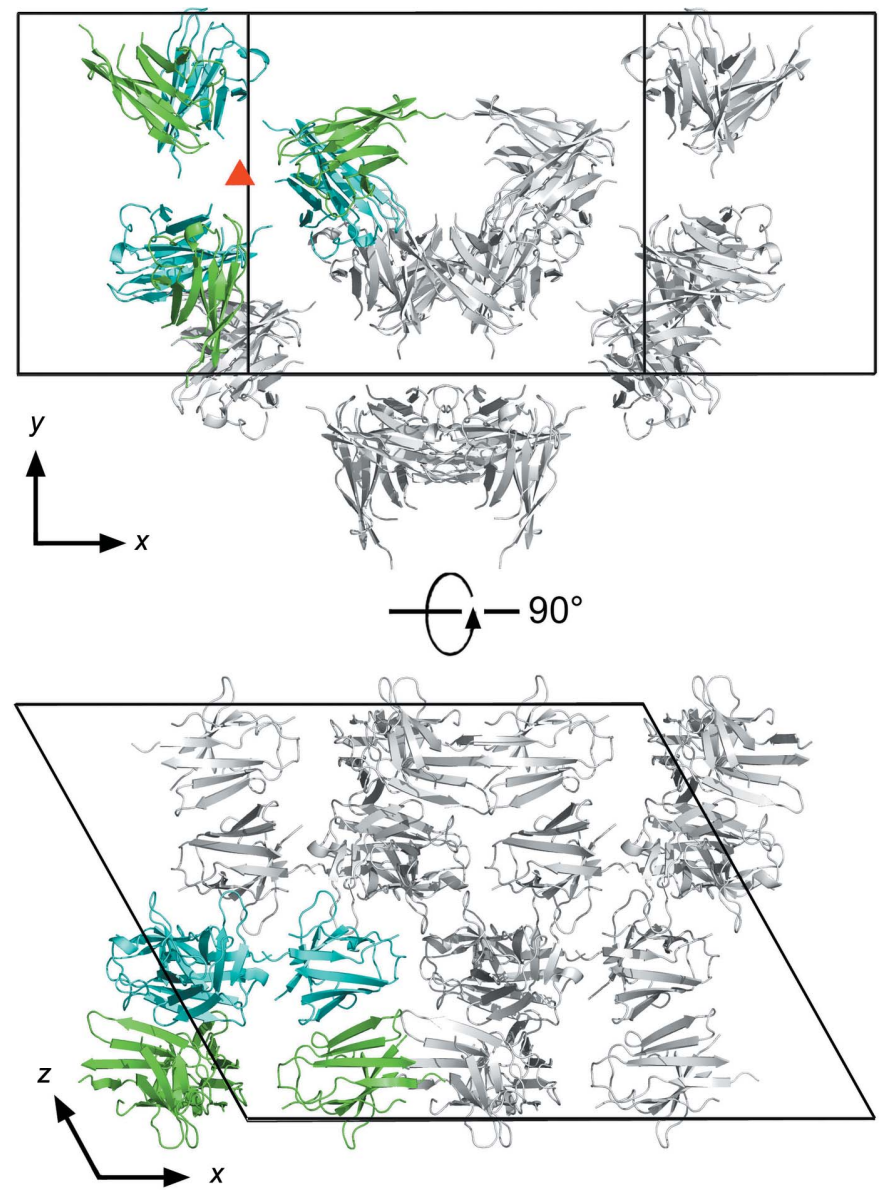

Figure 5

Crystal packing of Nb2907. Shown are two orientations of the unit cell of Nb2907 rotated $90^{\circ}$. The six nanobodies present in one asymmetric unit are coloured while the symmetry mates that complete the unit cell are shown in grey. The local threefold axis perpendicular to the $x-y$ plane is indicated by a red triangle. One set of nanobodies related by this local threefold axis is shown in green, the other set in cyan. CDR loops (not present in the model) point towards the local threefold axis. 
lution (Fig. 4b). Calculation of Matthews coefficients suggest that either two or three $\mathrm{Nb} 2919$ molecules are present in the asymmetric unit with a Matthews coefficient of 3.16 or $2.11 \AA^{3} \mathrm{Da}^{-1}$ and a solvent content of 61.1 or $41.6 \%$, respectively. Molecular-replacement calculations yielded a solution consisting of two molecules in the asymmetric unit (LLG 619 and translation function $Z$-score 26.3). Again, solutions containing only a single or three molecules in the asymmetric unit scored significantly worse (LLG 170 and 356, and translation function $Z$-score 15.7 and 5.8, respectively).

Despite a 98\% sequence identity between Nb2907 and Nb2919, corresponding to a single amino-acid substitution in CDR1 and CDR2 and a single substitution in the framework region (Fig. 1), their crystals grow in different conditions and belong to different space groups. It is tempting to speculate that these amino acids help to dictate the crystal packing, in agreement with our molecularreplacement results. The resulting structures are contributing to the growing repertoire of CDR loop conformations of camelid $\mathrm{VHH}$ domains and will be used in later stages to obtain more insight into VAR2CSA adhesion to the placenta, including structure determination of DBL6 $\varepsilon$-nanobody complexes.

Once the influence of the nanobodies on the interaction between DBL6 $\varepsilon$ and CSA/IgM has been determined, the structure of the DBL6 $\varepsilon$-nanobody complexes will help clarify how VAR2CSA binds to the placenta and elucidate the role of DBL6 $\varepsilon$ in the compact structure of VAR2CSA. These results will eventually open perspectives for further structural and functional studies on the VAR2CSA protein.

We thank N. Buys and K. Willibal for their technical support for nanobody screening and selection, and Dr A. Wohlkonig and Dr S. Soror for their assistance with data collection for Nb2919. This work was also supported by the Vlaams Interuniversitair Instituut voor Biotechnology (VIB), the Onderzoeksfonds of the Vrije Universiteit Brussel (OZR-VUB), the Fund for Scientific Research of Flanders
(FWO-Vlaanderen), the Institute for the Encouragement of Scientific Research and Innovation of Brussels (ISRIB) FWO-Vlaanderen and the Hercules Foundation. AV is a recipient of a PhD fellowship from FWO-Vlaanderen. The Labex GR-Ex is funded by the program 'Investissements d'avenir'. The authors acknowledge the use of the PROXIMA-1 beamline at SOLEIL (Gif-Sur-Yvette, France) and the X06DA beamline at SLS (Villigen, Switzerland).

\section{References}

Baranova, E., Fronzes, R., Garcia-Pino, A., Van Gerven, N., Papapostolou, D., Péhau-Arnaudet, G., Pardon, E., Steyaert, J., Howorka, S. \& Remaut, H. (2012). Nature (London), 487, 119-122.

Barfod, L., Dalgaard, M., Pleman, S., Ofori, M., Pleass, R. \& Hviid, L. (2011). Proc. Natl Acad. Sci. USA, 108, 12485-12490.

Battye, T. G. G., Kontogiannis, L., Johnson, O., Powell, H. R. \& Leslie, A. G. W. (2011). Acta Cryst. D67, 271-281.

Conrath, K., Vincke, C., Stijlemans, B., Schymkowitz, J., Decanniere, K., Wyns, L., Muyldermans, S. \& Loris, R. (2005). J. Mol. Biol. 350, 112-125.

Domanska, K., Vanderhaegen, S., Srinivasan, V., Pardon, E., Dupeux, F., Marquez, J., Giorgetti, S., Stoppini, M., Wyns, L., Bellotti, V. \& Steyaert, J. (2011). Proc. Natl Acad. Sci. USA, 108, 1314-1319.

Fried, M., Nosten, F., Brockman, A., Brabin, B. J. \& Duffy, P. E. (1998). Nature (London), 395, 851-852.

Kabsch, W. (2010). Acta Cryst. D66, 133-144.

McCoy, A. J., Grosse-Kunstleve, R. W., Adams, P. D., Winn, M. D., Storoni, L. C. \& Read, R. J. (2007). J. Appl. Cryst. 40, 658-674.

Matthews, B. W. (1968). J. Mol. Biol. 33, 491-497.

Rasti, N., Namusoke, F., Che, A., Chen, Q., Staalsoe, T., Klinkert, M., Mirembe, F., Kironde, F. \& Wahlgren, M. (2006). Proc. Natl Acad. Sci. USA, 103, 13795-13800.

Salanti, A., Dahlbäck, M., Turner, L., Nielsen, M. A., Barfod, L., Magistrado, P., Jensen, A. T., Lavstsen, T., Ofori, M. F., Marsh, K., Hviid, L. \& Theander, T. G. (2004). J. Exp. Med. 200, 1197-1203.

Srivastava, A. et al. (2010). Proc. Natl Acad. Sci. USA, 107, 4884-4889.

Steyaert, J. \& Kobilka, B. K. (2011). Curr. Opin. Struct. Biol. 21, 567-572.

Vagin, A. \& Teplyakov, A. (1997). J. Appl. Cryst. 30, 1022-1025.

Winn, M. D. et al. (2011). Acta Cryst. D67, 235-242. 